\title{
Expression Pattern of Genes in Condyloma Acuminata Treated with Clinacanthus nutans Lindau Cream versus Podophyllin
}

\author{
Jiraporn Jantaravinid $\triangle$, , ${ }^{1}$ Sukhum Jiamton $\mathbb{D}^{2},{ }^{2}$ Chatchawan Srisawat $\left(\mathbb{D},{ }^{1}\right.$ \\ Bhoom Suktitipat $\mathbb{D}^{1,3}$ and Napatara Tirawanchai $\mathbb{D}^{1}$ \\ ${ }^{1}$ Department of Biochemistry, Faculty of Medicine Siriraj Hospital, Mahidol University, Bangkok 10700, Thailand \\ ${ }^{2}$ Department of Dermatology, Faculty of Medicine Siriraj Hospital, Mahidol University, Bangkok 10700, Thailand \\ ${ }^{3}$ Integrative Computational BioScience Center, Mahidol University, Nakhon Prathom 73170, Thailand
}

Correspondence should be addressed to Napatara Tirawanchai; nednapis.tir@mahidol.edu

Received 1 February 2021; Revised 25 August 2021; Accepted 31 August 2021; Published 17 September 2021

Academic Editor: Shuibin Lin

Copyright (c) 2021 Jiraporn Jantaravinid et al. This is an open access article distributed under the Creative Commons Attribution License, which permits unrestricted use, distribution, and reproduction in any medium, provided the original work is properly cited.

\begin{abstract}
Clinacanthus nutans Lindua (C. nutans), a strong antiviral traditional medicine, can be used to treat condyloma acuminata (CA) caused by the human papillomavirus (HPV). However, its molecular mechanism for CA elimination is unknown. Herein, we conducted a randomized clinical trial to evaluate the effectiveness of $C$. nutans and its molecular mechanism compared with podophyllin, the gold standard treatment. Using a randomized block design, six patients were treated with $C$. nutans and podophyllin for four weeks. Efficacy of drugs was assessed by size reduction of the warts and HPV viral load quantification using droplet digital PCR. The gene expression profiling of CA was analyzed using NanoString Technology. After the podophyllin and C. nutans treatments, CA lesion sizes were reduced to $97.0 \%$ and $84.4 \%$ clearance, and the HPV viral loads were reduced by $74.0 \%$ and $46.6 \%$, respectively. The gene expression pattern of immune profiling showed that 23 genes (i.e., HLA-DPB, CCL3, CXCL2, CXCR2, and OSM) were significantly differentially expressed by podophyllin, whereas 2 genes (IFNL1 and IRF2) were remarkably expressed by C. nutans. In inflammatory profiling, 108 genes (i.e., CXCL2, IL8, and STAT3) were highly expressed by podophyllin, but none of genes were observed to change expression by C. nutans. These results suggested that podophyllin may reduce the HPV infection through a mechanism related to proinflammatory response. In addition, C. nutans was found to suppress the HPV infection through mechanism related to the activation of immune response. This study shows novel therapeutic mechanisms of podophyllin and C. nutans. It is suggested that $C$. nutans might be used as an alternative treatment for CA treatment.
\end{abstract}

\section{Introduction}

Human papillomavirus (HPV) is etiologically associated with the development of genital warts. More than 100 different types of HPV have been classified as either low risk (LR-HPV) or high risk (HR-HPV), based on their oncogenic characteristics [1]. Condyloma acuminata (CA), or genital warts, are mainly attributable to the LR-HPV type $6(89 \%)$ and HPV type $11(11 \%)[2]$. Up to date, there are no antiviral drugs for HPV infection. Thus, therapeutic managements were not targeted at antiviral therapies but either attempt to remove physical lesion or stimulate immune response to eliminate viral infection [3]. Alternatively, medicinal plants have shown their potential to treat CA, such as podophyllin and polyphenon E (sinecatechins $15 \%$ ointment).

Clinacanthus nutans Lindau (C. nutans) is an important traditional medicine from Acanthaceae family that has been used as a vital medicine in tropical Asia. C. nutans has been proved to have anti-inflammatory, antioxidant, immune response and antiviral activities $[4,5]$. Furthermore, it has been reported that C. nutans compound inhibits HPV infections by interfering at the early step of an HPV16 infection, and it may have a TLR4transcriptional inducible effect, which was a key 
regulatory molecule to stimulate the immune response and inflammatory cytokines secretion [6]. Additionally, it is revealed that $C$. nutans could affect alteration in the nonspecific cell-mediate immune response, which relates to the releasing of IL4 from peripheral blood mononuclear cell [5]. In Thailand, C. nutans cream has been included in the National Lists of Essential Medicines for skin inflammations treatment from virus infection [7].

To date, efficacy of C. nutans cream for CA treatment has not yet been studied in clinical trials nor has molecular mechanisms been addressed. Hence, this study aims to investigate the efficacy of $C$. nutans treatment on HPVinfected CA patients in comparison with podophyllin. Size reduction of the warts together with the amount of HPV DNA and gene expression profiling in response to drug treatments were subjected in the study.

\section{Materials and Methods}

2.1. Patients. Six Thai male patients (aged $>18$ years) with clinical CA diagnosis at their first visit were enrolled into this study. Each patient must have at least two CA, with at least one centimeter apart between each wart. Patients had no medical history of immunomodulator or systematic antiviral drugs usage. Patients with documented HIV or autoimmune diseases were excluded. Written informed consent according to the Declaration of Helsinki approved by The Institution Review Board (IRB), Faculty of Medicine Siriraj Hospital, Mahidol University (COA no: $\mathrm{Si}$ 629/2016) were obtained from all patients and were registered in Thai Clinical Trials Registry (TRTC: 062875 7627).

2.2. Specimen and Sample Preparation. CA specimens were obtained from Dermatology Outpatients Unit at Siriraj Hospital, Mahidol University. Sample swabs were collected from both pretreatment and posttreatment visits. Samples were arranged in randomized block design. Each block was arranged in the block of four. Following random separation of the warts as "A" or "B," they were treated with C. nutans cream (The Government Pharmaceutical Organization, Thailand, GPO) or podophyllin (Vidhyasom, Thailand). Patients were followed up every week for one month, and sample swabs were collected at week 0 and week 4 . All sample swabs were preserved in DNA/RNA shield ${ }^{\mathrm{TM}}$ collection tubes at $-20^{\circ} \mathrm{C}$. Following extraction, DNA and RNA samples were subjected to HPV genotyping, HPV viral load, and gene expression profiling analysis.

2.3. Nucleic Acid Extraction. Genomic DNA and total RNA were extracted from the swab samples using the Allprep DNA/RNA Mini Kit (QIAGEN Ltd., UK). Concentration of the purified DNA was measured by a Nanodrop ${ }^{\mathrm{TM}} 8000$ spectrophotometer and stored at $-20^{\circ} \mathrm{C}$ until use. DNA samples with $260 / 280 \mathrm{~nm}$ absorbance ratio between 1.8 and 2.0 were diluted to approximately $5 \mathrm{ng} / \mu \mathrm{L}$ prior to ddPCR. RNA concentration was also measured, and its purity was approximately 2 by NanoPhotometer ${ }^{\circledR}$ before gene expression analysis was immediately performed.
2.4. Linear Array HPV Genotyping Assay. Linear array HPV genotyping assay was performed by the Molecular Microbiology Laboratory, Department of Microbiology, Faculty of Medicine Siriraj Hospital, Mahidol University using the linear array HPV genotyping test (Roche Diagnostics, Switzerland).

2.5. Droplet Digital PCR (ddPCR). HPV L1 gene was determined using QX200 ddPCR system (Bio-Rad) in comparison with human $\beta$-globin gene as a reference gene. Reactions were set up according to the manufacturer's instructions. All primers and ddPCR conditions were shown in Table S1. Using QX200 Droplet reader ${ }^{\mathrm{TM}}$, ddPCR droplets were analyzed by QuantaSoft ${ }^{\mathrm{TM}}$ analysis software (version 1.7.4.0917). Quantitative measurement represented the copy number $/ \mu \mathrm{L}$ of the PCR reaction for the HPV target gene in comparison with $\beta$-globin gene.

2.6. NanoString nCounter Gene Expression Assay and Data Analysis. Immune and inflammatory panels were selected for gene expression analysis using NanoString nCounter ${ }^{\circledR}$ Platform (NanoString Technology). The reactions were set up according to the nCounter XT Gene expression protocol. Gene expression analysis was performed using nSolver Analysis Software v4.0 (NanoString Technology) involving in 770 genes using immune panel (NS_CANCERIMMUNE_V1.1) and a custom designed set of 256 genes in inflammatory panel (NS_INFL_HS_V2_C2534). A background correction was made by subtracting the background thresholds of negative control from raw counts. Adjusted raw counts were normalized using the combination of positive controls and reference gene normalization. The positive control normalization was computed by the geometric mean normalization factor, whereas reference gene normalization was calculated by the geometric mean of 11 genes, SF3A3, MRP55, TLK2, HDAC3, PPIA, TBP, ZNF143, DNAJC14, ABCF1 SAP130, and EDC3, in immune panel and four genes, CLTC, CUSB, HPRT1, and PGK1, in inflammatory panel.

2.7. Statistical Analysis. SPSS Statistic version 18 Software (IBM, USA) was used for clinical data and the viral load measurement. Pre- and posttreatments using podophyllin or C. nutans were compared by nonparametric Wilcoxon signed-rank test. Results were considered significant at $p$ value $<0.05$. Data on gene expression assay were normalized using nSolver 4.0 according to the manufacturer recommendations. Two-tailed Student's $t$ tests were used to evaluate significant differences in gene expression between pre- and posttreatments using podophyllin and C. nutans. The $p$-values were adjusted using false discovery rate (FDR) method for multiple comparison (Benjamini-Hochberg) $[8,9]$. FDRs less than 0.05 were considered significantly different. Volcano plot was created to depict differential gene expression between pre- and posttreatments on their original scale. Data were reported as a fold change in gene expression. 


\section{Results}

3.1. HPV Genotyping from CA Lesions. Prevalence of HPV genotypes from six patients revealed six different genotypes, which were classified into LR-HPV and HR-HPV. The most common HPV genotype was LR-HPV. Only one out of six patients had both LR-HPV and HR-HPV genotypes. Regarding to the pattern of the HPV infections, four patients (66.66\%) had single HPV genotype, whereas two patients (33.33\%) revealed multiple HPV genotypes. Of four patients with single HPV genotype, HPV-6 was found in three patients (75\%), whereas HPV-11 was found in only one patient (25\%). In contrast, two out of the six patients had multiple HPV genotypes. One patient had two HPV genotypes (HPV11 and HPV-40), and another had four HPV genotypes (HPV-11, HPV-81, HPV-45, and HPV-58). In case of multiple infections, HPV-11 was dominantly detected, with minor infection from other HPV genotypes. Thus, it is demonstrated that HPV-6 and HPV-11 were dominantly detected (Table 1).

\subsection{Clinical Outcomes and HPV Viral Load Clearance.} Baseline clinical outcome and HPV viral load clearances were described in Table 1. Five patients received podophyllin and $C$. nutans treatment were classified into 2 groups; responder (wart size and viral load were approximately $>75 \%$ reduction) and nonresponder (wart size and viral load were $<75 \%$ reduction). Based on the CA clearance, median of the total clearances for responder of podophyllin and C. nutans treatment groups were $97 \%$ and $84.85 \%$ size reduction area respectively $(p<0.05$, Wilcoxon signed-rank test, $n=5)$. However, one patient (ID 03), was classified as nonresponder showed that lesion were not completely cured.

It is shown from the HPV viral load quantification that medians of HPV viral load of the pre- and postpodophyllin treatments were 1.74 and 0.34 HPV copies/cell, respectively, in the responder group $(p=0.043$, Wilcoxon signed-rank test; $n=5)$. As to responder of $C$. nutans treatment, median values of HPV viral load were reduced from 1.74 to 0.71 HPV copies/cell, and difference was statistically significant ( $p=0.043$, Wilcoxon signed rank test, $n=5)$. For nonresponder group, which has viral load reduction less than $75 \%$, only one patient (ID03) showed a slight decrease in HPV viral load after podophyllin treatment, but the HPV viral load greatly increased after $C$. nutans treatment. The rates of HPV viral load clearances after podophyllin and C. nutans treatments were $74 \%$ and $46.58 \%$, respectively. These data were found to be significantly different $(p=0.043$; Wilcoxon signed-rank test, $n=5$ ) (Table 1 , Files S1).

3.3. Immune Gene Expression Profiling of CA after Podophyllin or C. nutans Treatments. It was observed from the hierarchical cluster analysis using expression ratio that patterns of the gene expression between pre- and posttreatment of podophyllin and $C$. nutans were significantly different (Figure 1). In the podophyllin treatment group, there were 23 significantly differentially expressed genes identified by volcano plot filtering (adjusted $p<0.05$; fold change >1.2) (Figure S1). Among them, two genes were upregulated and 21 genes were downregulated (Table S2). It is suggested that efficacy of podophyllin treatment might affect expression of the immune genes, for example cytokine, cell function, regulation, antigen processing, pathogen defense, TNF Superfamily, and cytotoxic gene sets. For C. nutans treatment group, there were only two differentially expressed genes identified by the volcano plot filtering (adjusted $p<0.05$; fold change $>2$ ) (Figure S2). These two genes were upregulated, and no gene was downregulated (Table S3). Changing in the state from before C. nutans treatment to after $C$. nutans treatment was observed as lessseparated cluster by differences in expression profiles of genes. These results demonstrated that efficacy of $C$. nutans treatment might affect only on some genes relating to cytokine and chemokine.

Furthermore, the differentially expressed genes in the podophyllin treatment group were compared with those in the C. nutans treatment group using Venn diagram (File S2). At posttreatment, 23 genes were differentially expressed for podophyllin, and only 3 genes for C. nutans. Interestingly, only IFNL1 gene was upregulated in both podophyllin and C. nutans treatments. Additionally, $H L A-D P B 1$ gene was shown to be significantly upregulated in podophyllin treatment, whereas IFNL1, IRF2 genes were found to be significantly upregulated in $C$. nutans treatment. On the contrary, 21 genes were found to be downregulated in podophyllin treatment only.

\subsection{Inflammatory Gene Expression Profiling of CA after} Podophyllin or C. nutans Treatments. It was demonstrated from the heatmap hierarchical clustering that there was a differential expression of genes in the inflammatory pathway between pre- and posttreatment of both drugs (Figure 2).

Surprisingly, it was identified from the volcano plot filtering that 108 genes were significantly upregulated in podophyllin-treated CA (adjusted $p<0.05$; fold change $>1.20$ ) (Figure S3, Table S4). This implied that podophyllin might affect the expression of genes involved in inflammatory pathway. In C. nutans-treated group, 58 genes were shown to be differentially expressed in a volcano plot filtering (adjusted $p \leq 0.10$; fold change $>1.20$ ). However, none of the inflammatory genes were found to be differentially expressed if $p<0.05$ was adjusted (Figure S4, Table S5). It is observed that the expression profiles of those genes were not significantly different between pre- and post-C. nutans treatments. Hence, it is suggested that $C$. nutans might not affect the inflammatory response pathway.

\section{Discussion}

HPV is etiologically associated with the development of virtually all CA. It is widely accepted that HPV-6 and HPV11 are dominant LR-HPV in the pathogenesis of CA [10]. Our results showed that the most common types of HPV identified in CA lesions were HPV-6 and HPV-11, which was consistent with the previous studies $[11,12]$. However, 


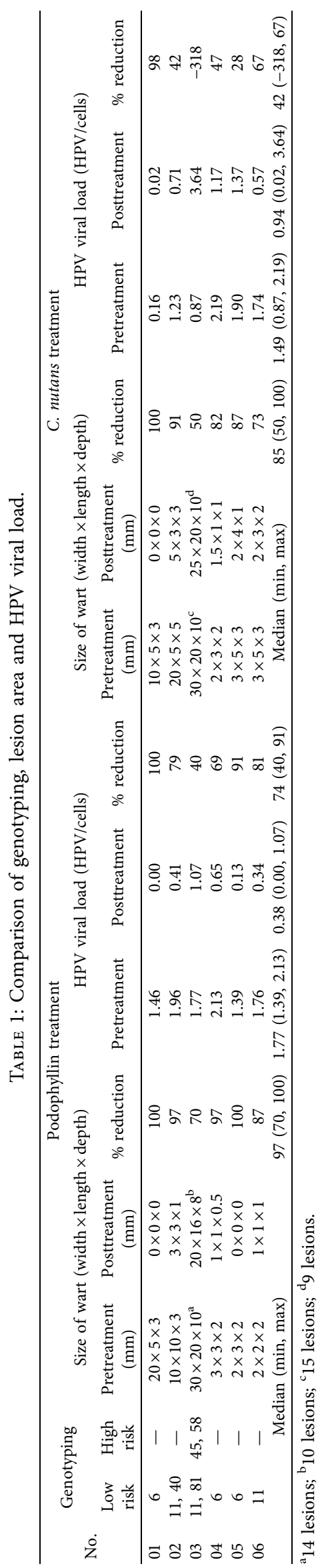




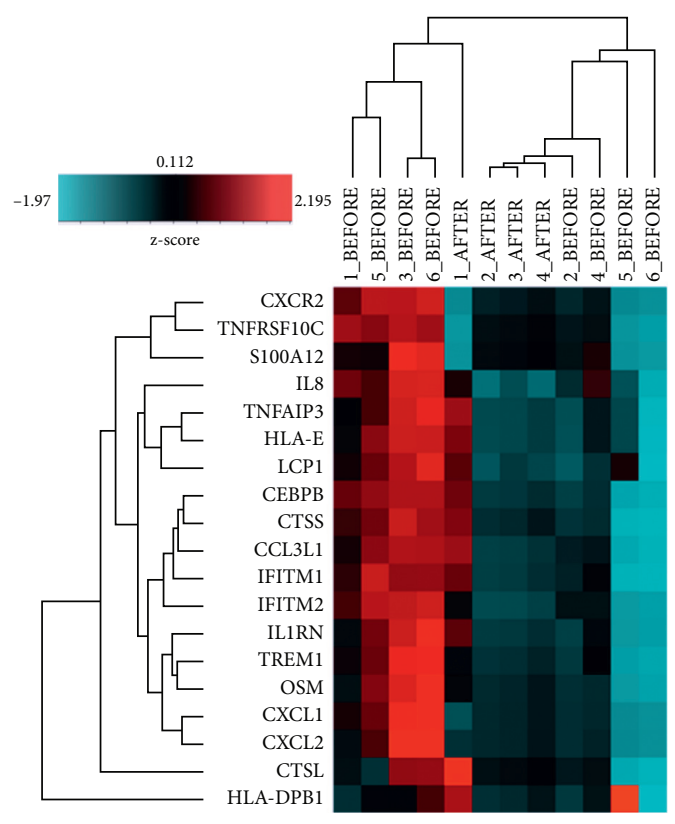

(a)

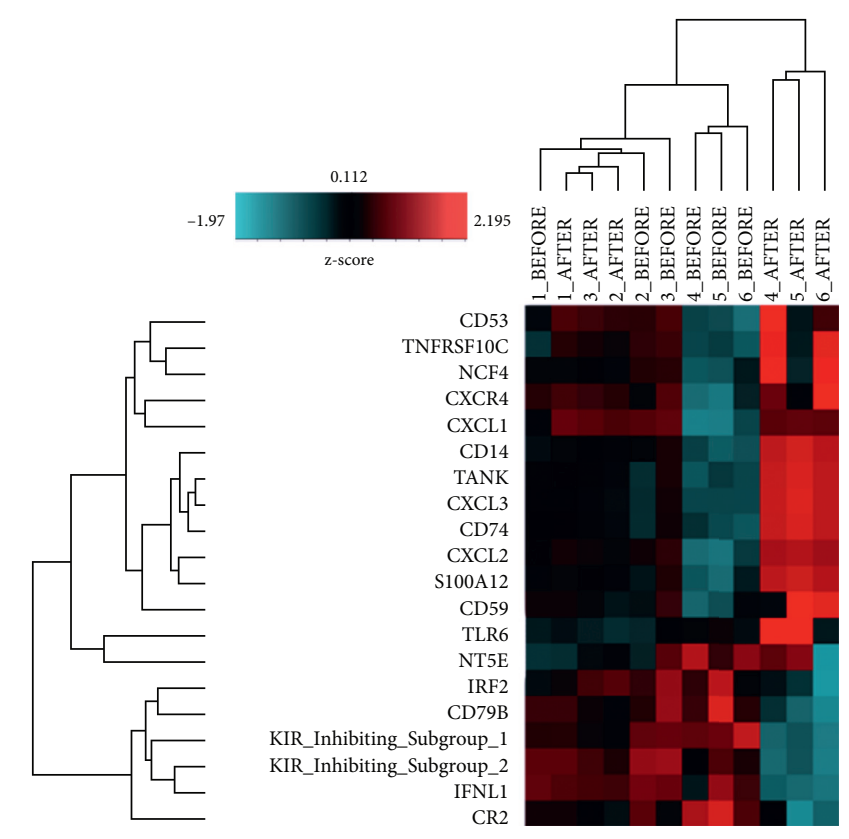

(b)

FIGURE 1: Immune gene profiling of pre- and post-treatment group as measured by NanoString Technology. (A) Heatmap of significantly altered gene expression levels by Z-score of podophyllin treatment. (B) Heatmap of significantly altered gene expression levels by Z-score of C. nutans treatment. The number horizontal bar along top is annotated by the patient samples. The red color indicates an increases in the expression of genes, and the blue color indicates a decrease in gene expression.

multiple infection was also detected in some CA lesions (ID02 and ID03).

Podophyllin has been successfully utilized as a treatment for CA patients, and it has been found to be effective in reducing superficial CA [13]. In randomized controlled trials, podophyllin treatment yields moderate clearance rates of $41 \%-47 \%$, while recurrent rates were found to be around $25 \%-70 \%$ [13]. However, there are adverse effects of podophyllin, such as long-term toxicity, mutagenicity, and carcinogenesis [14]. Interestingly, C. nutans showed compelling effects in preclinical in vitro and in vivo studies, suggesting that it has multifaceted properties, involving in antiviral, anti-inflammation and antiproliferative activities [15]. Evidence from the meta-analysis of randomized controlled trial studies demonstrated that C. nutans cream is useful and has high efficacy in Herpes genitalis treatment [16]. In Thailand, $4 \%$ C. nutans cream (w/w) is commercially available, and it is recommended to apply 4 times daily [17]. However, there has been no medication recommendation for C. nutans-treated CA. This is the first study to evaluate the clinical effectiveness of $C$. nutans cream in a well-controlled clinical design. It was found that clearance rate of podophyllin treatment was similar to those observed by other clinical studies [18]. Interestingly, high clearance rate $(84.85 \%)$ was found using C. nutans in LR-HPV-infected CA, but low clearance rate (50\%) was found in HR-HPVinfected CA. These results suggested that $C$. nutans might be more effective in the clearance of LR-HPV infection than HR-HPV infection. In summary, podophyllin showed $74 \%$ reduction of HPV viral load in all patients, which was consistent with 97\% CA lesion clearance, whereas C. nutans was able to reduce the HPV viral load for $42 \%$, which was also consistent with $85 \%$ CA lesion clearance. These results were supported by our efficacy treatment analysis in that podophyllin cauterization was more effective than C. nutans. According to the $C$. nutans treatment, five of the six patients showed reduction in the HPV viral load in correlation with their CA lesion clearance. Only one patient (ID03) revealed high viral load even if the CA lesion was reduced, suggesting that $C$. nutans may not cure CA infected with HR-HPV.

Regardless of the efficacy treatment, there was a correlation between HPV genotyping and HPV viral load clearance. Podophyllin demonstrated significantly reduced viral load for both LR-HPV and HR-HPV, whereas C. nutans was found to reduce only LR-HPV viral load. Our results were consistent with other studies that podophyllin could effectively eliminate wart lesions from all HPV genotypes, resulting in no HPV detection [19]. As to the C. nutans, Sookmai et al. reported that C. nutans compounds could inhibit HPV infections in an in vitro study [6]. Therefore, it is suggested that podophyllin is an appropriate treatment for multiple HPV infections. On the other hand, C. nutans was less effective than podophyllin; hence, it is a suitable treatment for a single HPV infection and LR-HPV infection.

The immune response in HPV-infected CA is considered to be the main determinant of CA disease and its associated immunomodulatory approaches, which have gained attention as promising strategies for CA treatment. Therefore, this is the first report on the mechanism of action associated with the immune response after podophyllin and C. nutans treatment. To define the target 
genes, differentially expressed genes were screened by the volcano plot method. From total of 23 genes, two genes were upregulated and 21 were downregulated after podophyllin treatment. Only $H L A-D P B 1$ was significantly upregulated, whereas OSM, IL8, CXCL1, CXCL2, and CXCR2 were the five most significantly downregulated genes. Interestingly, $H L A-D P B 1$ was found to be upregulated after podophyllin treatment, which was a novel finding. HLA-DPB1 belongs to HLA (human leukocyte antigen) class II, which is associated with a major histocompatibility complex in viral pathogenesis [20]. It is explained that $D R B 1^{*} 14$, a polymorphism of HLA class II, confers a protection against cervical cancer [21]. It is speculated in our findings that upregulation of the genes involving in immune response might allow a permissive environment for viral invasion and replication. Furthermore, the upregulation of OSM gene was statistically significant observed. The OSM gene, Oncostatin M, an $I L$ 6 family cytokine, helps to promote cell senescence and inhibits growth [22]. Previously, it was reported that $O S M$ mRNA level was significantly upregulated in the HPV16-E6 but downregulated in the HPV negative group [23]. This is consistent with our results, which indicated that OSM downregulation occurred with the podophyllin treatment. Therefore, podophyllin may inhibit or downregulate OSM gene due to the HPV infection clearance. Surprisingly, the expression levels of IL 8 and CXCR2 genes were significantly downregulated. $I L 8$ is an important regulator of the innate immunity; it is also known as a potent proinflammatory cytokine that exerts its effects through binding to its G-protein-coupled receptor CXCR1 and CXCR2 [24]. Waugh et al. reported that inhibition of IL8-CXCR1/CXCR2 has been shown to have therapeutic potential for a variety of solid tumors [25]. We suggested that IL8 and CXCR2 pathway may serve as a novel mechanism after podophyllin treatment. After C. nutans treatment, IFNL1 and IRF2 were found to be significantly upregulated, while none of other genes were found to be significantly downregulated. Interestingly, IFNL1 or Interferon Lambda 1 stimulates clearance of norovirus infection independently of adaptive immune system, suggesting that innate immune responses may have a role in determining viral persistence [26]. Furthermore, IFNL1 treatment was reported to decrease HSV-2 shedding from the vaginal mucosa in in vivo study [27]. Hence, it is speculated that upregulation of the IFNL 1 by $C$. nutans treatment, which would lead to the activation of antiviral state in epithelial cell, was a critical first-line defense against pathogenic viruses. Interferon regulatory factor 2, IRF2, has emerged as a complex mediator of gene expression and cell cycle control [28]. Lace et al. reported an increase in HPV-E7 expression, which could downregulate the IRF2 promotor, displaying a complex negative feedback mechanism in response to signal transduction pathways [29]. This corresponds with our finding that upregulation of the IRF2 gene would lead to elimination of the HPV infection. It is assumed that an increased immunosurveillance in CA lesions may allow the virus clearance with C. nutans treatment (Figure 3).
Surprisingly, 108 genes associated with inflammatory process were significantly downregulated with the podophyllin treatment (Figure S3), whereas none of those genes were found to be upregulated or downregulated after the C. nutans treatment (Figure S4). All of downregulated genes were found to be possibly associated with the proinflammatory cytokines, chemokines, and receptors synthetic pathways. In this study, CCL3, CXCL2, CXCL1, IL8, and STAT3 were found to be the five most significantly downregulated genes. A significant downregulation of the genes was shown in response to a decreased expression of CCL3, CXCL1, and CXCL2. It is suggested that podophyllin might induce a negative feedback on the proinflammatory pathway, leading to a decrease in the size of lesions. Downregulation of STAT3 after podophyllin treatment was also a novel finding. STAT3, a signal transducer and activator of transcription 3, modulates the transcription of the genes involved in the regulation of cell differentiation, proliferation, and immune responses. Frank et al. revealed that a selective inhibitor of STAT3 signaling pathway could be used as an anticancer drug [30]. We suggested that downregulation of STAT3 after podophyllin treatment may be associated with the inhibition of HPV proliferation due to lesion and viral load clearance (Figure 3).

In addition, the gene expression profiling associated with immune and inflammation panels of podophyllin and C. nutans-treated CA were compared. Some differences were found in immunomic profiling after podophyllin and C. nutans treatments. With podophyllin treatment, most genes involved in immune and inflammatory response were downregulated, whereas with the $C$. nutans treatment, those genes were upregulated. These results suggested that podophyllin and C. nutans may operate different mechanisms for HPV clearance. Downregulation of genes involving in immune and inflammatory response after podophyllin treatment suggested that its mechanism of action may not be correlated with the immune response; however, it may be related to previously described antiproliferative activity leading to CA clearance [31]. It is speculated that $C$. nutans might not inhibit HPV replication directly. It is demonstrated that $C$. nutans could stimulate the production of cytokines; hence, increase the immune response, thereby diminishing $\mathrm{CA}$ and decreasing virus erosion [5]. Furthermore, downregulation of inflammatory genes might suggest that HPV clearance mechanisms of both drugs may be associated with anti-inflammatory signaling pathway as described previously [4]. These findings suggested that podophyllin can exert more potent anti-inflammatory response than C. nutans. Alternatively, HPV clearance mechanism of $C$. nutans might not be involved in the inflammation signaling pathway. It is speculated that some other genes might play a crosstalk between the immune response and inflammation signaling pathways leading to HPV clearance by podophyllin or $C$. nutans. These shared molecular mechanisms may extend a potential target for a more effectively treatment of CA.

Nevertheless, there are some limitations of this study. Firstly, long-term follow-up more than 1 year was not completed. Secondly, a number of HPV-infected subjects 


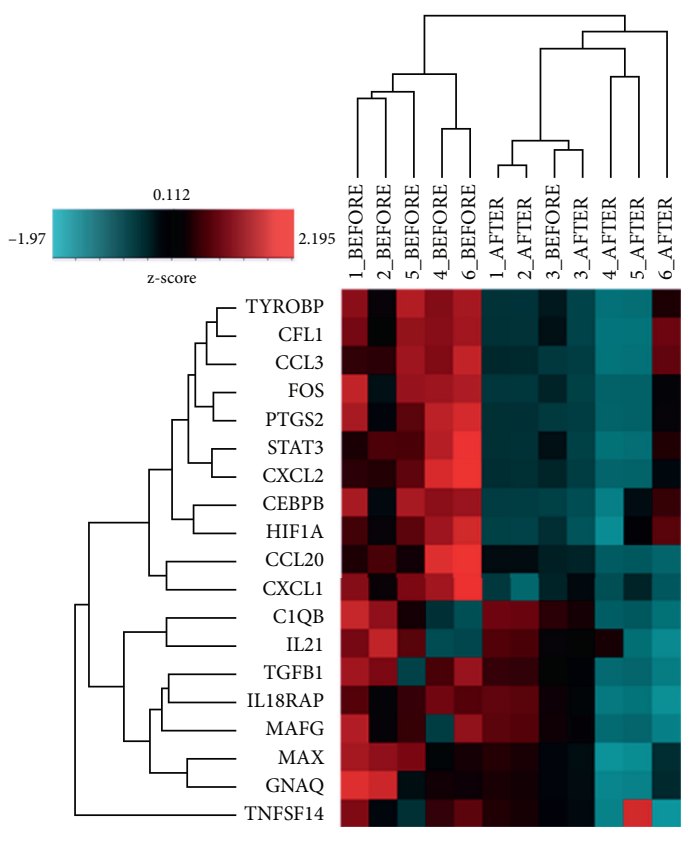

(a)

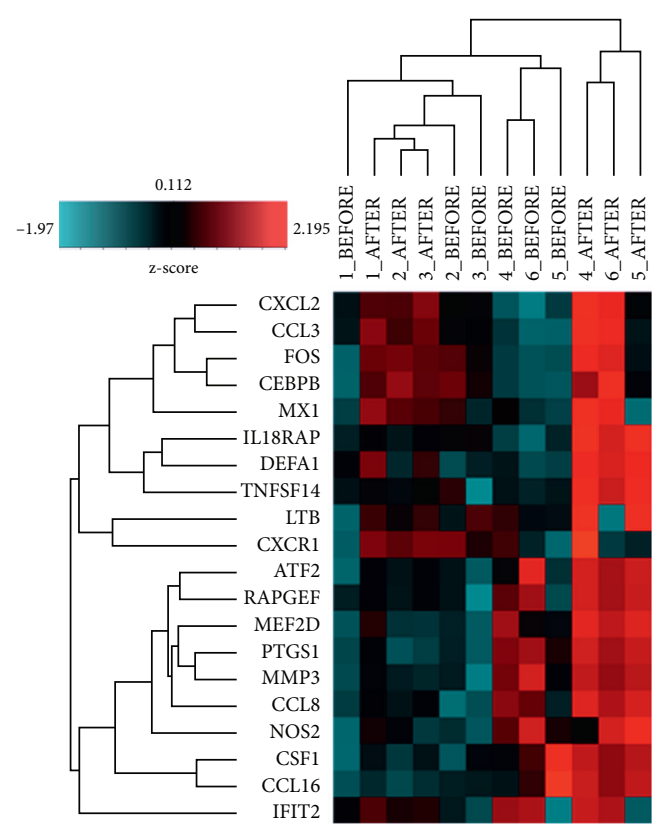

(b)

FIGURE 2: Inflammatory gene profiling of pre- and post-treatment group as measured by NanoString technology. (a) Heatmap of significantly altered gene expression levels by Z-score in of podophyllin treatment. (b) Heatmap of altered gene expression levels by Z-score of C. nutans treatment. The number horizontal bar along top is annotated by the patient samples. The red color indicates an increases in the expression of genes, and the blue color indicates a decrease in gene expression.

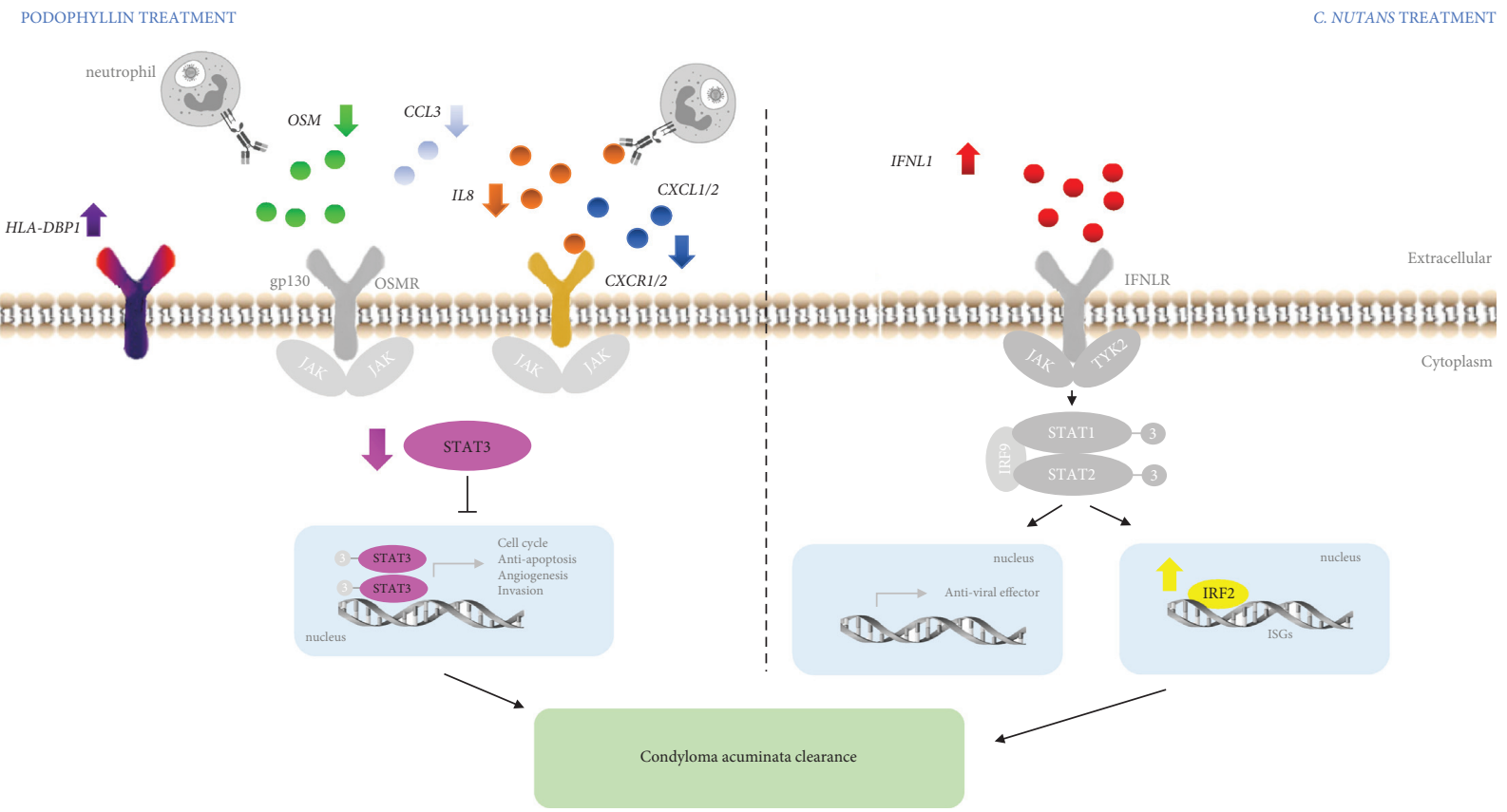

Figure 3: Proposed mechanism for podophyllin and C. nutans treatment. Various genes involving in inflammatory and immune response signaling were displayed. Gene cascade either be activated or inhibited, resulting in condyloma acuminata clearance.

was limited. Thirdly, heterogeneity leads to result uncertainty. Additionally, it is necessary to do further study on the molecular regulatory mechanism and other protein expression analysis of both drugs trials in order to identify therapeutic regimen. In turn, this may provide a guideline for the outcome on combination of these drugs for the CA treatment and indicate the underlying biological targets of CA in the future. However, this study might shed some light on applying C. nutans as an alternative treatment for HPVrelated CA with less long-term toxic effect. 


\section{Conclusion}

This study provides sufficient findings to demonstrate that the clinical efficacy and mechanism of action of podophyllin and $C$. nutans were, at least in part, due to reducing the inflammatory and modulating immune response signaling. Given the above findings, podophyllin possibly diminished HPV via both immune response and inflammatory signaling pathways, thereby allowing CA clearance. In contrast, C. nutans may promote the immune response while dampening inflammatory signaling, thereby given an overall tendency toward CA clearance.

\section{Data Availability}

The data used to support the findings of this study are included within the supplementary information files.

\section{Conflicts of Interest}

The authors declare that there are no conflicts of interest regarding the publication of this article.

\section{Acknowledgments}

The authors would like to thank Prof. Dr. Suthiluk Patumraj, Assoc. Prof. Dr. Sumate Ampawong, and Dr. Mudcharee Julotok for a kind discussion, Dr. Pattriya Chanyachailert, Dr. Yanin Nokdhes, Dr. Paramin Patthamalai, and $\mathrm{Mr}$. Viboon Omcharoen for kind assistance in patient recruitment and sample collection, the patients that participated in this study, the molecular genetics laboratory, Research Development, Faculty of Medicine Siriraj Hospital for providing NanoString Technology equipment, and Ms. Nerisa Thornsri for statistical analysis. This work was supported by Siriraj Research Fund (RO16034015), Faculty of Medicine Siriraj Hospital, Mahidol University, Thailand.

\section{Supplementary Materials}

Table S1: oligonucleotide primers and thermal cycling condition of HPV and $\beta$-globin gene. Table S2: top 20 differentially expressed gene in pre- and postpodophyllin treatments. Table S3: top 20 differentially expressed gene of pre- and post-C. nutans treatment in immune panel. Table S4: top 20 differentially expressed genes in inflammatory panel from pre- and postpodophyllin treatments. Table S5: top 20 differentially expressed gene of pre- and postC. nutans treatment in inflammatory panel. Figure S1: volcano plot showing differentially expressed genes in the immune panel from podophyllin treatment using NanoString Technology. Figure S2: volcano plot showing differentially expressed genes in the immune panel from C. nutans treatment using NanoString Technology. Figure S3: the volcano plot showing differentially expressed genes in the inflammatory panel from podophyllin treatment. Figure S4: volcano plot showing differentially expressed genes in the inflammatory panel from $C$. nutans treatment using NanoString Technology. File S1: (A) Clinical manifestation. (B) Agarose gel electrophoretic pattern of pre- and postdrug treatments. File S2: (A) Venn diagram of the number of gene expressions in the immune panels of the podophyllin and C. nutans treatment groups. (B) Top 20 differentially expressed genes from the podophyllin and C. nutans treated 24 CA samples in immune panel using NanoString Technology. (Supplementary Materials)

\section{References}

[1] M. A. Stanley, "Genital human papillomavirus infections: current and prospective therapies," Journal of General Virology, vol. 93, pp. 681-691, 2012.

[2] M. Grce and M. Mravak-Stipetić, "Human papillomavirusassociated diseases," Clinics in Dermatology, vol. 32, no. 2, pp. 253-258, 2014.

[3] M. Stanley, "Genital human papillomavirus infections--current and prospective therapies," JNCI Monographs, vol. 2003, no. 31, pp. 117-124, 2003.

[4] P. Wanikiat, A. Panthong, P. Sujayanon, C. Yoosook, A. G. Rossi, and V. Reutrakul, "The anti-inflammatory effects and the inhibition of neutrophil responsiveness by Barleria lupulina and Clinacanthus nutans extracts," Journal of Ethnopharmacology, vol. 116, no. 2, pp. 234-244, 2008.

[5] B. Sriwanthana, P. Chavalittumrong, and L. Chompuk, "Effect of Clinacanthus nutans on human cell-mediated immune response in vitro," Thai Journal of Pharmaceutical Sciences, vol. 20, no. 4, pp. 261-267, 1996.

[6] W. Sookmai, T. Ekalaksananan, C. Pientong, S. Sakdarat, and B. Kongyingyoes, "The anti-papillomavirus infectivity of Clinacanthus nutans compounds," Srinagarind Medical Journal, vol. 26, pp. 240-243, 2011.

[7] FDA National, List of Essential Medicines: Herbal Book, Ministry of Public Health, Thailand, 2012.

[8] M. Teufel, H. Seidel, K. Köchert, G. Meinhardt, R. S. Finn, and J. M. Llovet, "Biomarkers associated with response to regorafenib in patients with hepatocellular carcinoma," Gastroenterology, vol. 156, 2019.

[9] J. Ferreira and A. Zwinderman, "On the benjamini-hochberg method," The Annals of Statistics, vol. 34, no. 4, pp. 1827-1849, 2006.

[10] C. J. N. Lacey, C. M. Lowndes, and K. V. Shah, "Burden and management of non-cancerous HPV-related conditions: HPV-6/11 disease," Vaccine, vol. 24, pp. S35-S41, 2006.

[11] P. Leaungwutiwong, B. Bamrungsak, A. Jittmittraphap et al., "Molecular genotyping of human papillomavirus L1 gene in low-risk and high-risk populations in bangkok," Sexually Transmitted Diseases, vol. 42, no. 4, pp. 208-217, 2015.

[12] M. Skerlev, M. Grce, M. Sirotkoviæ-Skerlev, K. Husnjak, and J. Lipozenčić, "Human papillomavirus male genital infections: clinical variations and the significance of DNA typing," Clinics in Dermatology, vol. 20, no. 2, pp. 173-178, 2002.

[13] P. T. Ting and M. T. Dytoc, "Therapy of external anogenital warts and molluscum contagiosum: a literature review," Dermatologic Therapy, vol. 17, no. 1, pp. 68-101, 2004.

[14] G. Von Krogh and E. Longstaff, "Podophyllin office therapy against condyloma should be abandoned," Sexually Transmitted Infections, vol. 77, no. 6, pp. 409-412, 2001.

[15] A. Alam, S. Ferdosh, K. Ghafoor et al., "Clinacanthus nutans: a review of the medicinal uses, pharmacology and phytochemistry," Asian Pacific Journal of Tropical Medicine, vol. 9, no. 4, pp. 402-409, 2016.

[16] C. Kongkaew and N. Chaiyakunapruk, "Efficacy of Clinacanthus nutans extracts in patients with herpes infection: systematic review and meta-analysis of randomised clinical 
trials," Complementary Therapies in Medicine, vol. 19, no. 1, pp. 47-53, 2011.

[17] V. Thamlikitkul, "Treatment of herpes genitalis and herpes zoster with Clinacanthus nutans," Journal of Infectious Diseases and Antimicrobial Agents, vol. 12, pp. 95-96, 1995.

[18] J. Marcus and C. Camisa, "Podophyllin therapy for condyloma acuminatum," International Journal of Dermatology, vol. 29, no. 10, pp. 693-698, 1990.

[19] C. J. N. Lacey, R. L. Goodall, G. Ragnarson Tennvall et al., "Randomised controlled trial and economic evaluation of podophyllotoxin solution, podophyllotoxin cream, and podophyllin in the treatment of genital warts," Sexually Transmitted Infections, vol. 79, no. 4, pp. 270-275, 2003.

[20] C. Alves, T. Souza, I. Meyer, M. B. Toralles, and C. Brites, "Immunogenetics and infectious diseases: special reference to the mayor histocompatibility complex," Brazilian Journal of Infectious Diseases: An Official Publication of the Brazilian Society of Infectious Diseases, vol. 10, no. 2, pp. 122-131, 2006.

[21] Y. C. Yang, T. Y. Chang, Y. J. Lee et al., "HLA-DRB1 alleles and cervical squamous cell carcinoma: experimental study and meta-analysis," Human Immunology, vol. 67, no. 4-5, pp. 331-340, 2006.

[22] N. Finelt, A. Gazel, S. Gorelick, and M. Blumenberg, "Transcriptional responses of human epidermal keratinocytes to Oncostatin-M," Cytokine, vol. 31, no. 4, pp. 305-313, 2005.

[23] J. Chuerduangphui, C. Pientong, P. Chaiyarit et al., "Effect of human papillomavirus 16 oncoproteins on oncostatin $M$ upregulation in oral squamous cell carcinoma," Medical Oncology, vol. 33, no. 8, p. 83, 2016.

[24] H. U. Zeilhofer and W. Schorr, "Role of interleukin-8 in neutrophil signaling," Current Opinion in Hematology, vol. 7, no. 3, pp. 178-182, 2000.

[25] D. J. J. Waugh and C. Wilson, "The interleukin-8 pathway in cancer," Clinical Cancer Research, vol. 14, no. 21, pp. 6735-6741, 2008.

[26] T. J. Nice, M. T. Baldridge, B. T. McCune et al., "Interferon- $\lambda$ cures persistent murine norovirus infection in the absence of adaptive immunity," Science, vol. 347, no. 6219, pp. 269-273, 2015.

[27] N. Ank, H. West, C. Bartholdy, K. Eriksson, A. R. Thomsen, and S. R. Paludan, "Lambda Interferon (IFN- $\lambda$ ), a type III IFN, is induced by viruses and IFNs and displays potent antiviral activity against select virus infections in vivo," Journal of Virology, vol. 80, no. 9, pp. 4501-4509, 2006.

[28] S. Pettersson, M. Kelleher, E. Pion, M. Wallace, and K. L. Ball, "Role of Mdm2 acid domain interactions in recognition and ubiquitination of the transcription factor IRF-2," Biochemical Journal, vol. 418, no. 3, pp. 575-585, 2009.

[29] M. J. Lace, J. R. Anson, T. H. Haugen, and L. P. Turek, "Interferon regulatory factor (IRF)-2 activates the HPV-16 E6-E7 promoter in keratinocytes," Virology, vol. 399, no. 2, pp. 270-279, 2010.

[30] D. A. Frank, "STAT3 as a central mediator of neoplastic cellular transformation," Cancer Letters, vol. 251, no. 2, pp. 199-210, 2007.

[31] E. Longstaff and G. Von Krogh, "Condyloma eradication: selftherapy with $0.15-0.5 \%$ podophyllotoxin versus $20-25 \%$ podophyllin preparations-an integrated safety assessment," Regulatory Toxicology and Pharmacology, vol. 33, no. 2, pp. 117-137, 2001. 